\title{
Optimal weighted matchings for rank-deficient sparse matrices
}

\section{JD Hogg, JA Scott}

July 2012 
RAL Library

STFC Rutherford Appleton Laboratory

R61

Harwell Oxford

Didcot

OX11 0QX

Tel: $+44(0) 1235445384$

Fax: $+44(0) 1235446403$

email: libraryral@stfc.ac.uk

Science and Technology Facilities Council preprints are available online at: http:// epubs.stfc.ac.uk

ISSN 1361- 4762

Neither the Council nor the Laboratory accept any responsibility for loss or damage arising from the use of information contained in any of their reports or in any communication about their tests or investigations. 


\title{
Optimal weighted matchings for rank-deficient sparse matrices
}

\author{
J. D. Hogg J. A. Scott
}

July 10, 2012

\begin{abstract}
The maximum matching of a sparse matrix $A$ that maximizes the product of the matched entries can be used to increase the speed and reliability of sparse linear algebra operations. One popular method of solution is to transform to an assignment problem and to use a sparse variant of the Hungarian algorithm. If $A$ is structurally rank deficient this approach chooses a set $\mathcal{I} \times \mathcal{J}$ of rows and columns such that the restriction of $A$ to $\mathcal{I} \times \mathcal{I}$ is non-singular but, in general, the chosen $\mathcal{I}$ is sub-optimal. In this paper, we propose modifying the approach to obtain an optimal $\mathcal{I}$. We focus on the symmetric case and present results for rank-deficient sparse symmetric matrices arising from practical applications.
\end{abstract}

\section{Introduction}

The use of weighted matchings for the scaling of unsymmetric matrices is described by Duff and Koster [4] and, together with the symmetric adaption originally proposed by Duff and Gilbert [3] and subsequently built upon by Duff and Pralet [5], has been widely adopted by the sparse linear algebra community. Of particular note is the code MC64 from the HSL Mathematical Software Library [6] that implements the work of [4] and [5].

The core problem is as follows: given an $n \times n$ matrix $A=\left\{a_{i j}\right\}$, find a matching of the rows to the columns such that the product of the matched entries is maximised. That is, find a permutation $\sigma=\{\sigma(i)\}$ that solves the maximum product matching problem

$$
\max _{\sigma} \prod_{i=1}^{n}\left|a_{i \sigma(i)}\right|
$$

If $A$ is structurally rank deficient with rank $r<n$, this problem is ill-posed because the objective takes the value 0 for all possible $\sigma$. In this case, an ideal algorithm should return a matching on $\mathcal{I} \times \mathcal{J}$ (where $\mathcal{I}$ is a subset of the rows and $\mathcal{J}$ is a subset of the columns) of cardinality $r$ that solves the problem

$$
\max _{\mathcal{I}:|\mathcal{I}|=r} \max _{\sigma} \prod_{i \in \mathcal{I}}\left|a_{i \sigma(i)}\right|
$$

The algorithm proposed by Duff and Koster (which is summarised in Section 2) fails to do this. Instead, it returns a matching of cardinality $r$ that is optimal on a submatrix of $A$. This is demonstrated in Figure 1.1. Here and elsewhere, matrix entries are circled to indicate they belong to a matching.

In this paper, we consider the structurally rank-deficient case and, in particular, the symmetric structurally rank-deficient case. This is of interest because sparse symmetric indefinite linear systems that are structurally rank deficient can arise in a range of practical applications (including, for example, optimization). Duff and Pralet [5] also considered this case. In particular, they proved that if $A$ is symmetric and there is a maximum matching of $A$ on $\mathcal{I} \times \mathcal{J}$ then $A_{\mathcal{I} \times \mathcal{I}}$ (the restriction of $A$ to $\mathcal{I} \times \mathcal{I}$ ) is structurally non-singular. Thus there is a maximum matching for $A_{\mathcal{I} \times \mathcal{I}}$ of cardinality $r$ and this can be used to obtain a matching for $A$. However, their approach may lead to a sub-optimal matching (that is, the choice of $\mathcal{I}$ may be sub-optimal). The aim of this paper is to propose a modified approach that leads to an optimal solution. 
Figure 1.1: Example demonstrating the sub-optimality of the Duff and Koster algorithm on a symmetric structurally rank-deficient matrix. Matched entries are circled.

$\begin{array}{lllll}1 & 2 & 3 & 4 & 5\end{array}$

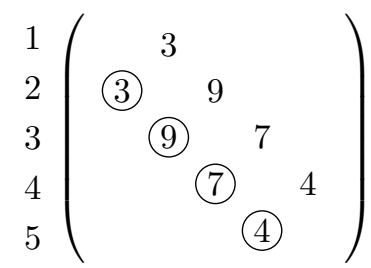

Duff and Koster matching $\begin{array}{lllll}1 & 2 & 3 & 4 & 5\end{array}$

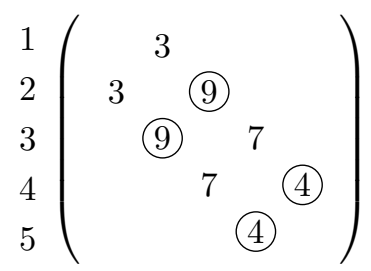

Optimal matching

This paper is organised as follows. We end this section by introducing notation and terminology that we use in the remainder of the paper. The original algorithm of Duff and Koster is briefly described in Section 2. We discuss the extensions by Duff and Pralet for symmetric matrices (including structurally singular matrices) in Section 3. In Section 4, a new preprocessing is proposed for symmetric matrices. Modifications to the Duff and Koster algorithm for structurally rank-deficient matrices are discussed in Section 5; this is the key contribution of this paper. An analysis of our algorithmic modification when applied to symmetric problems from practical applications is presented in Section 6. Finally, some concluding remarks are made in Section 7.

\subsection{Notation and terminology}

Let $A=\left\{a_{i j}\right\}$ be a general $n \times n$ sparse matrix. With $A$ we associate a bipartite graph $\mathcal{G}_{A}=\left(V_{\text {row }} \cup V_{\text {col }}, E\right)$. The node sets $V_{\text {row }}=\left\{\right.$ row $_{1}$, row $_{2}, \ldots$, row $\left._{n}\right\}$ and $V_{\text {col }}=\left\{\operatorname{col}_{1}, \operatorname{col}_{2}, \ldots, \operatorname{col}_{n}\right\}$ correspond to the rows and columns of $A$; an edge $\left(\operatorname{row}_{i}, \operatorname{col}_{j}\right)$ belongs to $E$ if and only if $a_{i j} \neq 0$. An edge $\left(\operatorname{row}_{i}, \operatorname{col}_{j}\right) \in E$ is said to be incident on nodes row $_{i}$ and $\mathrm{col}_{j}$.

An edge subset $\mathcal{M} \subseteq E$ is called a matching (or assignment) if no two edges in $\mathcal{M}$ are incident to the same node. In matrix terms, a matching corresponds to a set of nonzero entries with no two in the same row or column. A node is matched if there is an edge in the matching incident on the node, and to be unmatched otherwise. We denote by $\mathcal{M}^{\prime}$ the transpose matching $\left\{\left(\operatorname{row}_{j}, \operatorname{col}_{i}\right) \mid\left(\operatorname{row}_{i}, \operatorname{col}_{j}\right) \in \mathcal{M}\right\}$.

The cardinality of a matching is the number of edges in it. A maximum cardinality matching (or maximum matching) is a matching of maximum cardinality (this is $n$ if $A$ is structurally non-singular). If $A$ is symmetric and $\mathcal{M}$ is a maximum matching in $\mathcal{G}_{A}$ then, because of symmetry, $\mathcal{M}^{\prime}$ is also of maximum cardinality and has the set of row indices $V_{c o l}$ and the set of column indices $V_{\text {row }}$.

A path $\mathcal{P}$ in $\mathcal{G}_{A}$ is an ordered set of edges in which successive edges are incident to the same node. $\mathcal{P}$ is called an $\mathcal{M}$-alternating path if the edges of $\mathcal{P}$ are alternately in $\mathcal{M}$ and not in $\mathcal{M}$. An $\mathcal{M}$-alternating path is an $\mathcal{M}$-augmenting path if it connects an unmatched column node with an unmatched row node.

Let $\mathcal{M}$ and $\mathcal{P}$ be subsets of $E$ and define

$$
\mathcal{M} \oplus \mathcal{P}:=(\mathcal{M} \backslash \mathcal{P}) \cup(\mathcal{P} \backslash \mathcal{M}) .
$$

If $\mathcal{M}$ is a matching and $\mathcal{P}$ is an $\mathcal{M}$-augmenting path, then $\mathcal{M} \oplus \mathcal{P}$ is again a matching and $\mathcal{M} \oplus \mathcal{P}=|\mathcal{M}|+1$.

Let $W=\left\{w_{i j}\right\}$ be a real-valued $n \times n$ sparse matrix with $w_{i j} \geq 0$. Let $\mathcal{G}_{W}=\left(V_{\text {row }}, V_{\text {col }}, E\right)$ be the corresponding bipartite graph each of whose edges $\left(\right.$ row $\left._{i}, \operatorname{col}_{j}\right) \in E$ has weight $w_{i j}$. The weight $w(\mathcal{M})$ of a matching $\mathcal{M}$ in $\mathcal{G}_{W}$ is the sum of its edge weights, that is,

$$
w(\mathcal{M})=\sum_{\left(\text {row }_{i}, \text { col }_{j}\right) \in \mathcal{M}} w_{i j} .
$$

An $\mathcal{M}$-augmenting path $\mathcal{P}$ starting at an unmatched column node $j$ is called shortest if

$$
w(\mathcal{M} \oplus \mathcal{P}) \leq w(\mathcal{M} \oplus \tilde{\mathcal{P}})
$$


for all other possible $M$-augmenting paths $\tilde{\mathcal{P}}$ starting at node $j$. The length of an alternating path $\mathcal{P}$ is defined to be

$$
l(\mathcal{P}):=w(\mathcal{M} \oplus \mathcal{P})-w(\mathcal{M})=w(\mathcal{P} \backslash \mathcal{M})-w(\mathcal{M} \cap \mathcal{P})
$$

In the following, if $\mathcal{M}$ is a matching, $\mathcal{I}$ and $\mathcal{J}$ denote the set of row and column indices matched by $\mathcal{M}$, i.e., $\mathcal{I}=\left\{i:\left(\operatorname{row}_{i}, \operatorname{col}_{j}\right) \in \mathcal{M}\right.$ for some $\left.j\right\}$ and $\mathcal{J}=\left\{j:\left(\operatorname{row}_{i}, \operatorname{col}_{j}\right) \in \mathcal{M}\right.$ for some $\left.i\right\}$. The matrix formed by selecting the rows and columns indexed by $\mathcal{I}$ and $\mathcal{J}$, respectively, is called the submatrix of $A$ restricted to the rows in $\mathcal{I}$ and the columns in $\mathcal{J}$ and is denoted by $A_{\mathcal{I} \times \mathcal{J}}$.

$\mathcal{M}$ corresponds to a column permutation $\sigma=\{\sigma(i)\}$; it is convenient to also refer to $\sigma$ as a matching. $\mathcal{M}$ is called a symmetric matching if and only if $j=\sigma(i) \Rightarrow i=\sigma(j)$.

\section{The Duff and Koster Algorithm}

As we explain in this section, the algorithm of Duff and Koster transforms the maximum product matching problem to an assignment problem that can be solved with minimal modifications to standard methods.

Let $c_{j}=\max _{i}\left|a_{i j}\right|$ be the maximum absolute value in column $j$ of $A$. Define a preprocessing of $A$ to $\hat{A}=\left\{\hat{a}_{i j}\right\}$ as follows:

$$
\hat{a}_{i j}= \begin{cases}\log c_{j}-\log \left|a_{i j}\right|, & \text { for } a_{i j} \neq 0 \\ \infty & \text { otherwise }\end{cases}
$$

The linear sum assignment problem

$$
\min \sum_{i=1}^{n} \hat{a}_{i \sigma(i)}
$$

has the same optimal permutation as the maximum product matching problem (1.1). Further, if there exists variables $u_{i}$ and $v_{j}$ with

$$
\hat{a}_{i j}-u_{i}-v_{j} \begin{cases}=0, & \text { for } j=\sigma(i), \\ \geq 0, & \text { otherwise }\end{cases}
$$

then $u_{i}$ and $v_{j}$ are optimal dual variables for (2.2). The dual solution may be used to scale the matrix [8] by defining diagonal matrices $S^{r}$ and $S^{c}$ with entries

$$
\begin{aligned}
s_{i}^{r} & =\exp \left(u_{i}\right), \\
s_{j}^{c} & =\exp \left(v_{j}-\log c_{j}\right) .
\end{aligned}
$$

The scaled matrix $S^{r} A S^{c}$ has matched entries that are one in absolute value with all other entries less than or equal to one in absolute value.

The assignment problem can be solved efficiently using a variant of the Hungarian algorithm [7] outlined as Algorithm 1. The algorithm can be initialized by setting $\mathcal{I}=\phi, \mathcal{J}=\phi, u=0, v=0$, although in practice it is warm-started. For full rank matrices, each iteration of the algorithm adds an additional row $i$ to $\mathcal{I}$ and an additional column $j$ to $\mathcal{J}$ and updates the matching so that it is optimal on the new $\mathcal{I} \times \mathcal{J}$. If the matrix is structurally rank deficient, the cardinality of the final matching will be $r<n$, where $r$ is the rank of $A$. The matching is optimal on $A_{\mathcal{I} \times \mathcal{J}}$, but may be sub-optimal on $A$ (that is, another choice of $\mathcal{I} \times \mathcal{J}$ could result in increasing (1.2)).

The set $\mathcal{I} \times \mathcal{J}$ is extended by selecting an unmatched column $j$ and then finding the shortest $\sigma$-augmenting path $\mathcal{P}$ from $j$ to an unmatched row $i$. In matrix terms, an alternating path starts from the unmatched column $j$, and proceeds through zero or more pairs of entries $\left(\hat{a}_{t k}, \hat{a}_{t \sigma(t)}\right)$ to subsequent columns before terminating at an unmatched row. The edge weights are given by $\hat{a}_{t \sigma(t)}-u_{t}-v_{\sigma(t)}$. The Hungarian algorithm is essentially the repeated application of a sparse version of Dijkstra's algorithm [2] to accomplish the task of extending a matching on a column-by-column basis.

Once $\mathcal{P}$ is found, the matching $\sigma$ is augmented along it, that is, all the unmatched entries on the path become matched entries, while the previously matched entries become unmatched. As the shortest 


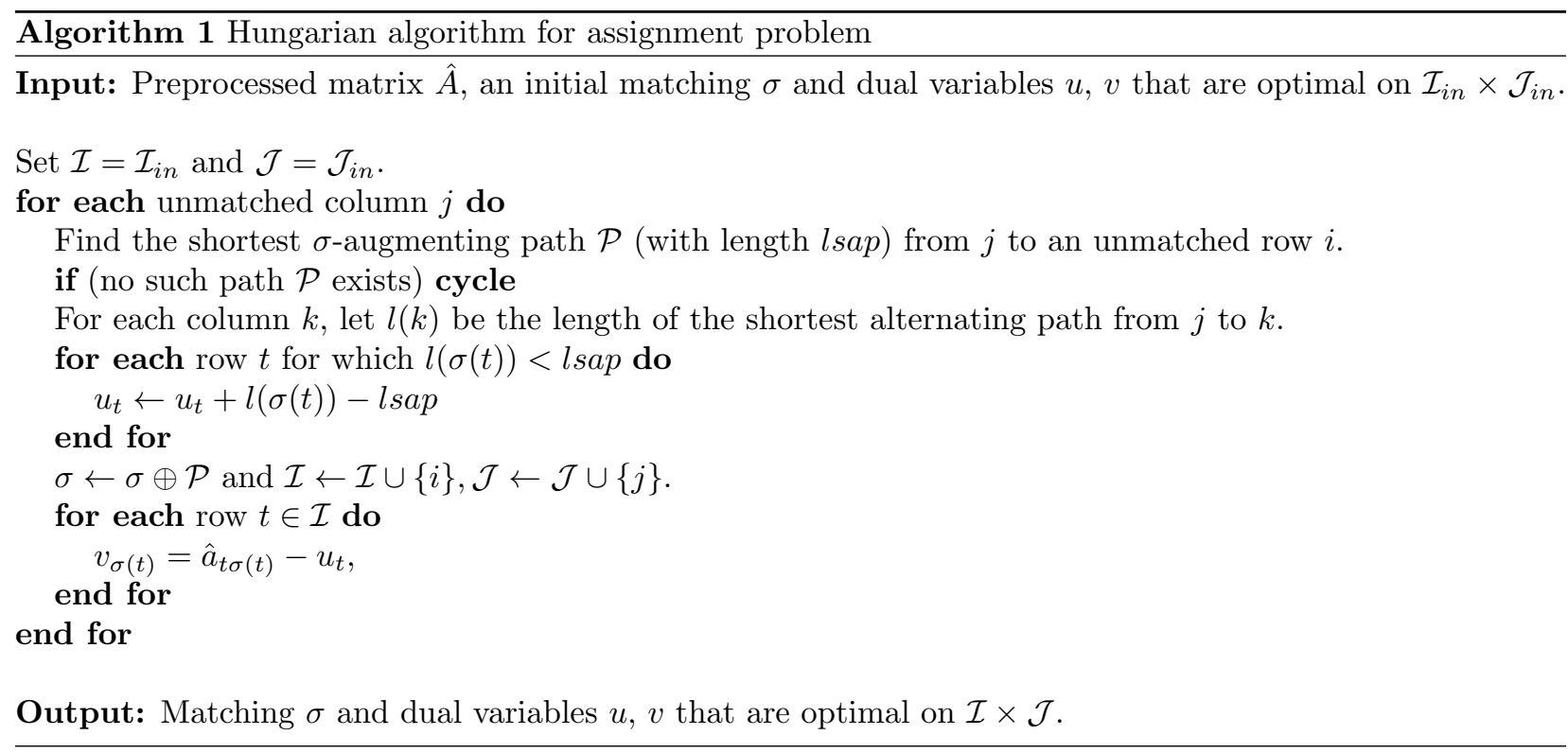

$\sigma$-augmented path was chosen, the new matching is optimal on the extended set $\tilde{\mathcal{I}} \times \tilde{\mathcal{J}}$ with $\tilde{\mathcal{I}}=\mathcal{I} \cup\{i\}$ and $\tilde{\mathcal{J}}=\mathcal{J} \cup\{j\}$.

The dual variables $u$ and $v$ must be updated for the new matching. Let $l s a p$ be the length of the shortest augmenting path and $l(k)$ be the length of the shortest alternating path from $j$ to column $k$. For each row $t$ for which $l(\sigma(t))<$ lsap, the update

$$
u_{t} \leftarrow u_{t}+l(\sigma(t))-l s a p
$$

is computed. Then for each $t \in \tilde{\mathcal{I}}$

$$
v_{\sigma(t)}=\hat{a}_{t \sigma(t)}-u_{t} .
$$

Further details may be found in [4]. For clarity, we summarise the complete Duff and Koster algorithm for unsymmetric matrices as Algorithm 2.

\begin{tabular}{l}
\hline Algorithm 2 Duff and Koster maximum weighted matching algorithm \\
\hline Input: Unsymmetric matrix $A$.
\end{tabular}

1. Apply the unsymmetric preprocessing (2.1) to $A$ to obtain $\hat{A}$.

2. Determine an initial matching $\sigma$ and dual variables $u, v$ that are optimal on $\mathcal{I}_{\text {in }} \times \mathcal{J}_{\text {in }}$.

3. Apply Algorithm 1 to $\hat{A}$.

Return the maximum matching and dual variables.

Compute the scaling factors (2.3).

Output: A column permutation (with unmatched columns flagged) and scaling factors for $A$.

\section{Duff and Pralet modification for symmetric matrices}

So far, we have described the matching algorithm for a general (unsymmetric) matrix. We now focus on the symmetric case. First, we observe that a symmetric matrix need not have a symmetric matching. To illustrate this, consider the following example: 


$$
\left(\begin{array}{lll} 
& 1 & 2 \\
1 & 3 \\
2 & 3 &
\end{array}\right)
$$

However, it is generally important when scaling a symmetric matrix that symmetry is preserved (i.e. the scaling factors (2.3) should satisfy $s=s^{r}=s^{c}$ ).

Duff and Pralet [5] show that if $A$ is non-singular, the geometric average of the row and column scalings is sufficient to maintain desirable properties. That is, they build a diagonal scaling matrix $S$ with entries

$$
s_{i}=\sqrt{s_{i}^{r} s_{i}^{c}},
$$

with $s_{i}^{r}$ and $s_{i}^{c}$ given by (2.3), and compute the symmetrically scaled matrix $S A S$. The entries in the scaled matrix that are in the matching have absolute value one while the rest have absolute value less than or equal to one.

For rank-deficient matrices, Duff and Pralet prove that, if Algorithm 1 returns a matching on $\mathcal{I} \times \mathcal{J}$ (with cardinality equal to the rank $r$ of $A$ ), $A_{\mathcal{I} \times \mathcal{I}}$ is structurally non-singular. A maximum matching for $A_{\mathcal{I} \times \mathcal{I}}$ is obtained by applying Algorithm 1 to $A_{\mathcal{I} \times \mathcal{I}}$ and then mapped back to a (sub-optimal) matching for $A$ (with $n-r$ rows and columns unmatched). If the scaling factors for $A_{\mathcal{I} \times \mathcal{I}}$ are $s_{i}^{r}$ and $s_{j}^{c}$, Duff and Pralet propose using scaling factors

$$
s_{i}= \begin{cases}\sqrt{s_{i}^{r} s_{i}^{c}} & \text { if } i \in \mathcal{I} \\ \frac{1}{\max _{k \in \mathcal{I}}\left|a_{i k} s_{k}\right|} & \text { otherwise }\end{cases}
$$

with the convection $1 / 0=1$. With this choice, the absolute values of the entries of $S A S$ are again less than or equal to one, with those in the matching having absolute value equal to one. Moreover, the entry of largest absolute value in each non-empty row/column is one.

The approach of Duff and Pralet is summarised in Algorithm 3.

Algorithm 3 Duff and Pralet variant for symmetric matrices

Input: An $n \times n$ symmetric matrix $A$.

1. Apply the unsymmetric preprocessing (2.1) to $A$ to obtain $\hat{A}$.

2. Determine an initial matching $\sigma$ and dual variables $u, v$ that are optimal on $\mathcal{I}_{\text {in }} \times \mathcal{J}_{\text {in }}$.

3. Apply Algorithm 1 to $\hat{A}$ to obtain a maximum matching on $\mathcal{I} \times \mathcal{J}$.

4. if $(|\mathcal{I}|=n)$ then

Compute the scaling factors (3.1). else

5. Apply Algorithm 1 to $A_{\mathcal{I} \times \mathcal{I}}$

Use the maximum matching for $A_{\mathcal{I} \times \mathcal{I}}$ to compute a matching for $A$.

Compute the scaling factors (3.2). end if

Output: A symmetric permutation (with unmatched rows/columns flagged) and scaling factors for $A$.

\section{$4 \quad$ Symmetric preprocessing}

If $A$ is symmetric, in general the preprocessing (2.1) results in an unsymmetric matrix $\hat{A}$. This is undesirable in the rank-deficient case. For example, consider the following rank-2 matrix, where the optimal solution to (1.2) has been circled 


$$
\left(\begin{array}{ll}
1.0 & 10^{3} \\
10^{3} & \\
10^{9} &
\end{array}\right) .
$$

Preprocessing (using $\log _{10}$ for convenience) gives

$$
\left(\begin{array}{lll}
9 & 0 & 0 \\
6 & & \\
0 & &
\end{array}\right)
$$

In this case it is impossible to distinguish columns 2 and 3 and Algorithm 1 matches columns 1 and 2 to rows 3 and 1 , respectively.

In the non-singular case, this loss of symmetry is not a problem since all the entries in each column are scaled and, by non-singularity, there is sufficient information retained to distinguish between the columns.

For symmetric matrices, to retain symmetry, we propose the following preprocessing

$$
\hat{a}_{i j}=\left(\log c_{j}+\log r_{i}\right) / 2-\log \left|a_{i j}\right|,
$$

where $r_{i}=\max _{j}\left|a_{i j}\right|$ is the maximum absolute value in row $i$ (observe $c_{i}=r_{i}$ for symmetric matrices).

Applying (4.1) to our example, Algorithm 1 returns the desired matching

$$
\left(\begin{array}{lll}
9 & 3 & 0 \\
3 & & \\
0 &
\end{array}\right) .
$$

Although for this simple case, restricting the matching to $\mathcal{I} \times \mathcal{I}$ would have been sufficient to obtain the better matching, this is not necessarily always the case. Thus we need to also consider modifying the matching algorithm for rank-deficient symmetric matrices and this is what we consider in the next section.

\section{Modified algorithm for rank-deficient matrices}

Recall the example from Figure 1.1: the reason that Algorithm 1 returns a sub-optimal matching is as follows. Column 5 is the last unmatched column to be considered, with the matching shown in Figure 1.1 already existing for columns 1 to 4 . There is no path between column 5 and row 1, so no augmenting path is found. Hence column 5 remains unmatched regardless of the values of the nonzero entries. A sub-optimal matching is only possible in the structurally rank-deficient case.

To motivate our proposed new approach, consider the application of Algorithm 1 to the following modified version of the example matrix. All the zeros have been replaced by a nonzero value $\epsilon$, a number so small that it will never be chosen as part of the matching if there is an alternative.

$$
\begin{aligned}
& 1 \\
& 2 \\
& 3 \\
& 4 \\
& 5
\end{aligned}\left(\begin{array}{ccccc}
1 & 2 & 3 & 4 & 5 \\
\epsilon & 3 & \epsilon & \epsilon & \epsilon \\
3 & \epsilon & 9 & \epsilon & \epsilon \\
\epsilon & 9 & \epsilon & 7 & \epsilon \\
\epsilon & \epsilon & 7 & \epsilon & 4 \\
\epsilon & \epsilon & \epsilon & 4 & \epsilon
\end{array}\right) .
$$


Figure 5.1: Different paths involving $\alpha$ entries

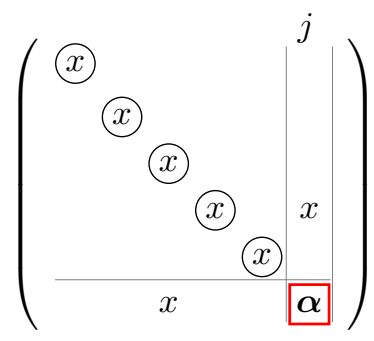

Case 1

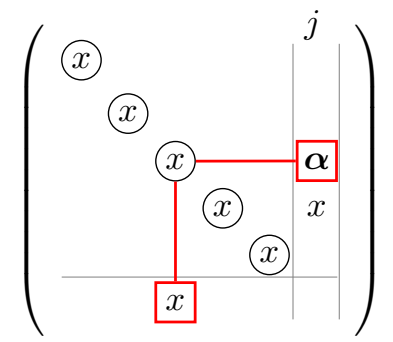

Case 2

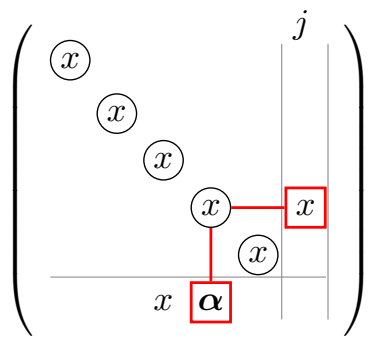

Case 3

By the optimality of Algorithm 1 for non-singular matrices, the computed matching shown is optimal. The rank-deficiency is apparent in that the matching contains an epsilon in the $(1,1)$ position. An optimal matching for the original rank-deficient matrix is obtained by restricting the matching to those rows and columns that are not matched on an epsilon.

This approach is not practical for real problems as the conversion from a sparse to a dense matrix increases the storage (and hence memory bandwidth) requirements to $O\left(n^{2}\right)$. However, by implementing the addition of the $\epsilon$ entries implicitly, the approach can be made workable and this is what we now explain.

Denote the modified matrix by $A(\epsilon)$. The preprocessing (4.1) transforms each $\epsilon$ entry to

$$
\hat{a}_{i j}(\epsilon)=\left(\log c_{j}+\log r_{i}\right) / 2+\alpha,
$$

where $\alpha$ is very large. Such entries can be calculated on the fly and an implicit representation of $\hat{A}(\epsilon)$ can be stored in the same space as $\hat{A}$ plus a vector of column maximums and a vector of row maximums (in the symmetric case, $c_{i}=r_{i}$ for all $i$ so a single vector is needed). An entry of $\hat{A}(\epsilon)$ that corresponds to an $\epsilon$ in $A(\epsilon)$ will be referred to as an $\alpha$ entry.

If a path exists from the current unmatched column to an unmatched row in the original matrix $A$ and the dual variables $u$ contain no term in $\alpha$, an $\alpha$ entry will never be included on the path $\mathcal{P}$ during the execution of Algorithm 1 ( $\alpha$ is too large for it to be included in a shortest path). The condition on $u$ is trivially satisfied if no path containing $\alpha$ has been augmented along (as $l$ sap $\ll \alpha$ ); thus it follows that $\alpha$ entries are never considered if $A$ is full rank and Algorithm 1 is applied to $\hat{A}(\epsilon)$.

When an unmatched column is found and there exists no path to an unmatched row in the original matrix, we start considering $\alpha$ entries. Observe that since $A$ is square, the existence of an unmatched column $j$ in $\hat{A}(\epsilon)$ implies the existence of an unmatched row $i$ and, as no path to $i$ exists in $A$, each of the entries in column $j$ that corresponds to an unmatched row must be $\alpha$ entry. Hence a trivial path consisting of a single entry exists with length $\alpha+\gamma$ where $|\gamma| \ll \alpha$. If the dual variables $u$ are much less than $\alpha$, we do not need to consider paths that contain more than one $\alpha$ entry since the lengths of such paths will be at least $2 \alpha$ (and hence greater than the length of the trivial path). There are three possibilities for paths with one $\alpha$ entry:

1. A trivial path is chosen using an $\alpha$ entry in column $j$ and an unmatched row.

2. An $\alpha$ entry in column $j$ and a currently matched row $i$ is chosen. There exists a path from column $\sigma(i)$ that is matched with row $i$ to an unmatched row.

3. An $\alpha$ entry in a column belonging to $\operatorname{Reach}(j)$ and an unmatched row is chosen, where $\operatorname{Reach}(j)$ is the set of columns $k$ such that a path exists from $j$ to $k$ in the original matrix $A$.

These are illustrated in Figure 5.1. Note that case 1 is a special case of case 3. We further observe that we can choose to augment along paths involving both cases 2 and 3 as they must be disjoint.

So far, we have assumed that the dual variables $u$ are much less than $\alpha$. This requires that there are no $\alpha$ entries in the current matching. If we were to relax this requirement, the storage needed for $u$ would double because a coefficient for $\alpha$ would have to be stored (merely using a large value results in loss of the precision 
needed to distinguish between candidate paths). Moreover, for some columns, all the entries (including the $\alpha$ entries) would need to be considered, and this means the full matrix would have to be explored each time such a column is encountered. This additional computation is not desirable and is prohibitive for large problems. To avoid this, we propose that rather than including the $\alpha$ entry in the matching, we augment along the path excluding the $\alpha$ value. The size of the matching will not increase but the optimal value will be improved. That is, once the algorithm starts considering the $\alpha$ entries, the cardinalities $|\mathcal{I}|$ and $|\mathcal{J}|$ remain constant, with one column (or row) swapped for another at each iteration. This may result in a suboptimal result. However, since an improvement in the objective value is made with each such augmentation, repeatedly iterating over unmatched columns until no further improvement is made will terminate with the optimal solution in a finite number of steps.

Let us consider the practical implementation further. For case 3, the set Reach $(j)$ and path lengths $l(k)$ are built in the process of determining that there is no path for the original matrix $\hat{A}$ from the unmatched column $j$ to an unmatched row. Since it does not matter which particular unmatched row $i$ the $\alpha$ entry belongs to (it will not be part of the augmenting path), contributions to the path length from $\log r_{i}$ and $u_{i}$ for the unmatched row can be ignored. We thus choose the column $k \in \operatorname{Reach}(j)$ for which $l(k)+\log \left(c_{k}\right) / 2-v_{k}$ is a minimum. The column set $\mathcal{J}$ is altered by removing $k$ and including $j$.

Case 2 requires considerably more work. For each matched row with matching column not in Reach $(j)$, we must explore possible paths that lead to an unmatched row and pick the shortest. This is very costly. However, observe that Algorithm 1 can be implemented row-wise rather than column-wise and case 2 in a row-wise implementation is covered by case 3 in a column-wise implementation. A row-wise implementation is equivalent to running the column-wise algorithm on $A^{T}$. The optimal solution can thus be found by applying Algorithm 1 alternately to $A$ and $A^{T}$ until the objective value ceases to decrease. In the symmetric case, $A=A^{T}$ and this can be exploited to save storage (provided symmetric preprocessing is used).

Based on our practical experience, we found it necessary to specify a minimum improvement (min_improv) to avoid cycling between two identical columns (or identical rows when running on $A^{T}$ ) because of floatingpoint inaccuracies. That is, we want to avoid at one step removing $k$ from the column set $\mathcal{J}$ and including $j$ and then, at the next step, removing $j$ and including $k$. We found it to be sufficient to require a minimum decrease of the square root of machine precision before altering $\mathcal{J}$. Using this, for each of the test problems used in Section 6, a single application to $A$ followed by one to $A^{T}$ yielded the optimal solution. This was unexpected and is not guaranteed (we are unable to prove that this should yield the optimal solution).

These modifications are summarised in our generalised Hungarian algorithm (which is for both symmetric and unsymmetric $A$ ), and in our proposed variant of the symmetric matrix matching and scaling algorithm (shown as Algorithms 4 and 5, respectively). If $A$ is structurally non-singular, Algorithm 4 reduces to Algorithm 1 but it differs in the action taken if no $\sigma$-augmenting path $\mathcal{P}$ is found and in the addition of a flag (change_flag) to indicate whether changes to $\sigma$ have occurred. In Algorithm 5, the generalised Hungarian algorithm is applied repeatedly to $A$ and $A^{T}$ until either the matching has cardinality $n$ or no changes to $\sigma$ are made. Then, in the rank-deficient case, we proceed as in the variant of Duff and Pralet to compute $A_{\mathcal{I} \times \mathcal{I}}$ and apply Algorithm 4 to it. The maximum matching obtained for $A_{\mathcal{I} \times \mathcal{I}}$ is finally mapped to a matching for $A$. We note that if $A$ is non-singular, a single application of the generalised Hungarian algorithm to $A$ gives a matching of cardinality $n$ and, in the rank-deficient case, a single application of the generalised Hungarian algorithm to $A_{\mathcal{I} \times \mathcal{I}}$ gives a matching of cardinality $r$. We further observe that when $A_{\mathcal{I} \times \mathcal{I}}$ is computed, $\mathcal{I}$ and $\mathcal{J}$ are optimal and so we can take the restriction of $A$ to $\mathcal{I} \times \mathcal{I}$ or to $\mathcal{J} \times \mathcal{J}$.

\section{$6 \quad$ Numerical experiments}

For our numerical experiments, we use two sets of symmetric structurally rank-deficient test problems. Both are taken from the University of Florida Sparse Matrix Collection [1]. Test Set 1 comprises 11 problems from the GHS_indef, Newman and Pajek groups; for this set we use the supplied values for the matrix entries. Test Set 2 consists of 8 problems that are structurally rank deficient but, because only the matrix pattern is available, we generate values for the entries. The generated values are distributed uniformally over the interval $[-1,1]$. Note that this means that the problems in Test Set 2, some of which are highly 


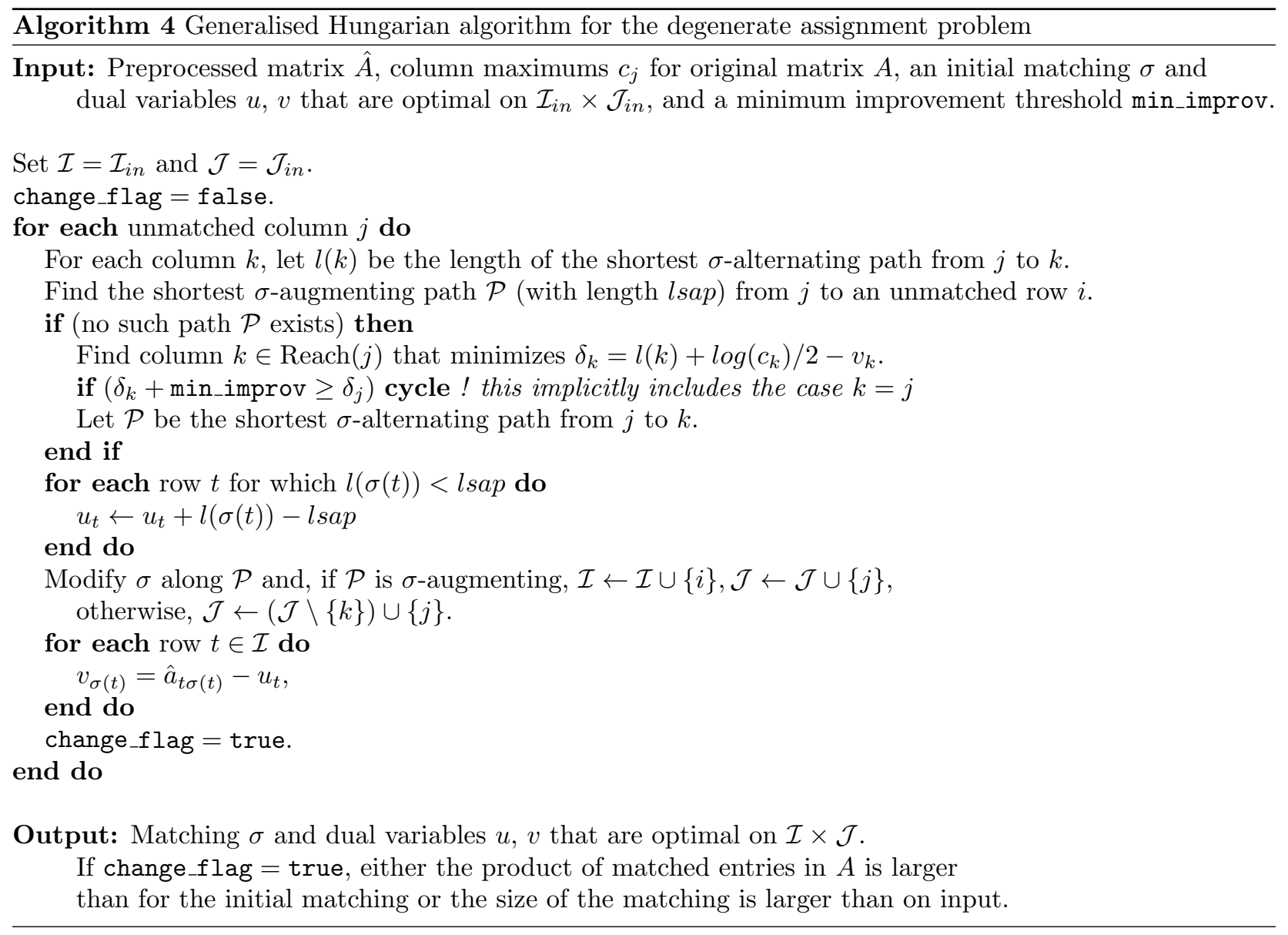




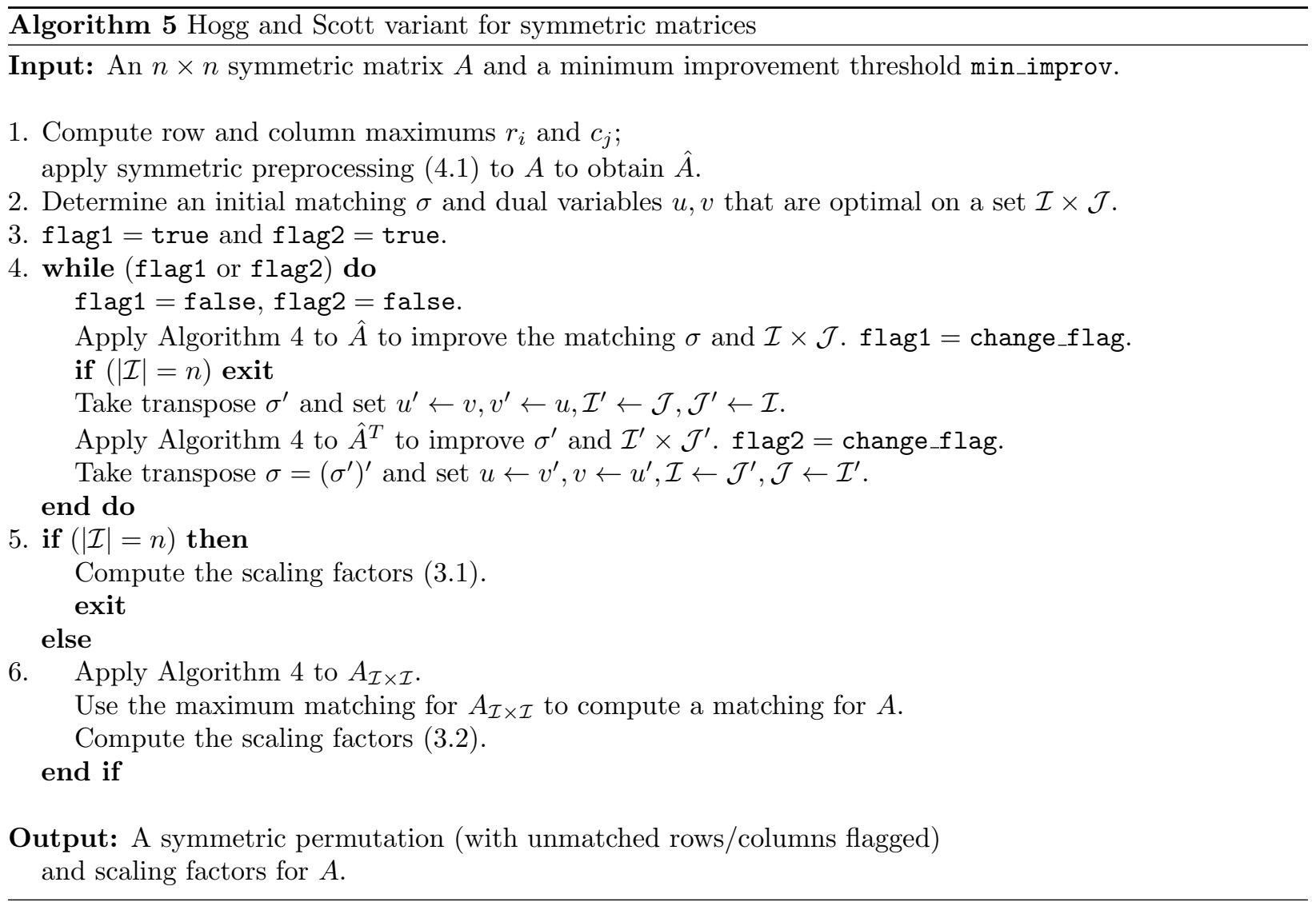


rank deficient, are well-scaled. We comment Duff and Pralet's focus in [5] was on more general indefinite matrices; their test set included only two rank-deficient problemsfor which $r=n-1$ and so they did not report on any highly rank-deficient matrices.

Table 6.1: Test Set 1: Symmetric rank-deficient matrices from practical problems. $n$ is the order of $A, n-r$ is the rank deficiency, and $n z$ is the number of entries in $A$.

\begin{tabular}{lrrr}
\hline Problem & \multicolumn{1}{c}{$n$} & $n-r$ & \multicolumn{1}{c}{$n z$} \\
\hline GHS_indef/aug2d & 29008 & $9800(33.8 \%)$ & 76832 \\
GHS_indef/aug2dc & 30200 & $10200(33.8 \%)$ & 80000 \\
GHS_indef/aug3d & 24300 & $12636(52.0 \%)$ & 69984 \\
GHS_indef/dtoc & 24993 & $4999(20.0 \%)$ & 69972 \\
Newman/astro-ph & 16706 & $990(5.92 \%)$ & 242502 \\
Newman/cond-mat & 16726 & $1130(6.76 \%)$ & 95188 \\
Newman/cond-mat-2003 & 31163 & $1989(6.38 \%)$ & 240058 \\
Newman/cond-mat-2005 & 40421 & $2453(6.07 \%)$ & 351382 \\
Newman/hep-th & 8361 & $1225(14.6 \%)$ & 31502 \\
Newman/netscience & 1589 & $165(10.4 \%)$ & 5484 \\
Pajek/Reuters911 & 13332 & $2647(19.9 \%)$ & 296076 \\
\hline
\end{tabular}

Table 6.2: Test Set 2: Symmetric rank-deficient matrices with patterns from practical problems and entries that are random values in $[-1,1] . n$ is the order of $A, n-r$ is the rank deficiency, and $n z$ is the number of entries in $A$.

\begin{tabular}{lrrr}
\hline Problem & \multicolumn{1}{c}{$n$} & \multicolumn{1}{c}{$n-r$} & \multicolumn{1}{c}{$n z$} \\
\hline AG-Monien/diag & 2559 & $169(6.60 \%)$ & 8184 \\
AG-Monien/netz4504 & 1961 & $617(31.5 \%)$ & 2342 \\
AG-Monien/stufe-10 & 24010 & $152(6.33 \%)$ & 92828 \\
AG-Monien/ukerbe1 & 5981 & $1871(31.3 \%)$ & 15704 \\
DIMACS10/kron_g500-logn16 & 65536 & $24076(36.7 \%)$ & 4912469 \\
Newman/as-22july06 & 22963 & $16362(71.2 \%)$ & 96872 \\
Pajek/Erdos981 & 485 & $58(12.0 \%)$ & 2762 \\
SNAP/Oregon-1 & 11492 & $8170(71.1 \%)$ & 46818 \\
\hline
\end{tabular}

The tests are performed on an INtel Xeon E5420 using the gfortran complier (version 4.4) with flags -O3. Our Fortran 95 implementations are built on the original implementation of Duff and Koster that is available within MC64. In all the tests, an initial matching $\sigma$ and dual variables $u, v$ that are optimal on a set $\mathcal{I} \times \mathcal{J}$ are computed in the same way (again following MC64).

\subsection{An analysis of different variants}

We are interested in assessing the quality of the computed ordering (and we omit computing the scaling factors). The statistic we use to measure the quality is

$$
\log \prod_{i \in \mathcal{I}}\left|a_{i \sigma(i)}\right|,
$$

which we refer to as the objective function value. The better the matching, the greater this value is. We report results for the algorithms discussed in this paper and for a number of variants of them. We first consider matchings that do not preserve symmetry and so do not employ the restriction $A_{\mathcal{I} \times \mathcal{I}}$ that imposes symmetry. We implement the following variants: 
I: Algorithm 2 (Duff and Koster).

II: Algorithm 2 with symmetric preprocessing.

III: Algorithm 5 with steps 5 and 6 omitted and, in step 4, only a single pass on $\hat{A}$. That is, step 4 is replaced by the following single statement:

4. Apply Algorithm 4 to $\hat{A}$ to improve the matching $\sigma$ and $\mathcal{I} \times \mathcal{J}$.

IV: Algorithm 5 with steps 5 and 6 omitted.

Results are given in columns 2 to 5 of Table 6.3 and timings are reported in Table 6.4. Variant IV computes the optimal value of the objective function and, while it is independent of the initial ordering of $A$, the other variants depend on the initial ordering. For each problem, we present three results. In the first line, we report the value of the objective function (6.1) for the supplied ordering. We then apply each variant to 10 random permutations of $A$ and, in the next two lines, we report the difference between the objective function value for the supplied ordering and the best and worst objective function value over the 10 permutations. Note that the reported timings are for the supplied ordering; if $A$ is permuted, the subsequent time for computing the matching can be more or less than that given in Table 6.4 (in our experiments, timings could reduce by about 50 per cent or conversely increase by as much as a factor of 2). The problems GHS_indef/aug2d, GHS_indef/aug2dc, and GHS_indef/aug3d are omitted from Table 6.3 since the values of the nonzero entries in these matrices are \pm 1 and so the objective function has value 0 in all cases.

Comparing variants I and II, we see that, for our test examples, symmetric preprocessing consistently computes a higher objective function value than unsymmetric preprocessing, but in both cases the objective function value can be far from optimal (for example, AG-Monien/stufe-10 and DIMACS10/kron_g500$\log 16)$.

A comparison of the results for variants III and IV demonstrates how the matching is improved by applying Algorithm 4 to both $A_{\mathcal{I} \times \mathcal{I}}$ and $A_{\mathcal{I} \times \mathcal{I}}^{T}$. For problems with only a small rank deficiency, the improvement is generally small, but where there is a larger rank deficiency (such as Newman/as-22july06 and DIMACS10/kron_g500-logn16), using both $A_{\mathcal{I} \times \mathcal{I}}$ and $A_{\mathcal{I} \times \mathcal{I}}^{T}$ can substantially increase the objective function value. Unfortunately, the cost of achieving this improvement can be significant. Moreover, we see that Algorithm 4 (used within III and IV) can be considerably more expensive than the simpler Algorithm 1 (used within I and II) (notable examples being Pajek/Reuters911 and DIMACS10/kron_g500-logn16).

It is worth noting that the additional steps added to create Algorithm 4 from Algorithm 1 account for only a small proportion of the additional runtime. Most of it is due to the modified algorithm exploring a larger number of columns. It is perhaps also appropriate to comment that the application of Algorithm 4 with a near-optimal input matching is likely to be inefficient. If an unmatched column is explored and no augmenting path is found then an efficient algorithm could cache the result gained from exploring those columns and use this information in subsequent searches. It is thus possible that with further work the time for variant IV could be brought much closer to that for variant III. However, this is outside the scope of the current study. 
Table 6.3: Comparison of the different variants. In each case, we give the objective function value (6.1) for the initial ordering of $A$ and the maximum increase and decrease in this value for 10 random permutations of $A$.

\begin{tabular}{|c|c|c|c|c|c|c|c|c|}
\hline Problem & I & II & III & IV & $\mathrm{V}$ & VI & VII & VIII \\
\hline \multirow[t]{3}{*}{ GHS_indef/dtoc } & -42577 & -42577 & -42577 & 0.00 & -85155 & -85155 & -42577 & 0.00 \\
\hline & $\begin{array}{r}+\quad 0.00\end{array}$ & 6746 & $\begin{array}{l}+16838 \\
\end{array}$ & 0.00 & $\begin{array}{l}+\quad 33677\end{array}$ & $\begin{array}{l}+33677\end{array}$ & $\begin{array}{l}+\quad 16838 \\
\end{array}$ & 0.00 \\
\hline & - $\quad 5783$ & 0.00 & 0.00 & 0.00 & 0.00 & 0.00 & 0.00 & 0.00 \\
\hline \multirow[t]{3}{*}{ Newman/astro-ph } & -15253 & -15202 & -15163 & -15090 & -15231 & -15236 & -15163 & -15090 \\
\hline & $\begin{array}{l}+5.59\end{array}$ & 0.00 & 6.88 & 0.00 & 4.63 & $+\quad 13.76$ & 6.88 & 0.00 \\
\hline & - 12.76 & - $\quad 18.77$ & 3.84 & 0.00 & $-\quad 15.48$ & 7.67 & 3.84 & 0.00 \\
\hline \multirow[t]{3}{*}{ Newman/cond-mat } & -10019 & -9870 & -9758 & -9635 & -9900 & -9880 & -9758 & -9635 \\
\hline & $+\quad 21.24$ & 0.00 & 0.00 & 0.00 & 0.00 & 0.00 & 0.00 & 0.00 \\
\hline & - 12.28 & - $\quad 56.09$ & $-\quad 42.52$ & 0.00 & - $\quad 75.14$ & - 85.04 & - 42.52 & 0.00 \\
\hline \multirow[t]{3}{*}{ Newman/cond-mat-2003 } & -21758 & -21571 & -21235 & -20961 & -21496 & -21508 & -21235 & -20961 \\
\hline & 0.00 & 0.00 & 0.00 & 0.00 & 0.00 & 0.00 & 0.00 & 0.00 \\
\hline & - 112.1 & - $\quad 120.4$ & - 84.85 & 0.00 & $-\quad 212.1$ & - $\quad 169.7$ & - 84.85 & 0.00 \\
\hline \multirow[t]{3}{*}{ Newman/cond-mat-2005 } & -29695 & -29449 & -29022 & -28673 & -29392 & -29370 & -29022 & -28673 \\
\hline & 0.00 & 0.00 & 0.00 & 0.00 & 0.00 & 0.00 & 0.00 & 0.00 \\
\hline & - $\quad 127.3$ & $-\quad 150.2$ & - $\quad 109.8$ & 0.00 & $-\quad 222.6$ & - 219.6 & - 109.8 & 0.00 \\
\hline \multirow[t]{3}{*}{ Newman/hep-th } & -1589 & -1490 & -1421 & -1320 & -1529 & -1522 & -1421 & -1320 \\
\hline & $+\quad 16.52$ & 0.00 & 0.00 & 0.00 & 0.00 & 0.00 & 0.00 & 0.00 \\
\hline & 6.87 & - 33.00 & $-\quad 17.83$ & 0.00 & $-\quad 35.98$ & $-\quad 35.67$ & - $\quad 17.83$ & 0.00 \\
\hline \multirow[t]{3}{*}{ Newman/netscience } & -1072 & -1066 & -1063 & -1059 & -1066 & -1067 & -1063 & -1059 \\
\hline & 3.87 & 0.00 & 0.88 & 0.00 & 0.00 & 1.75 & 0.88 & 0.00 \\
\hline & 3.18 & 3.79 & 1.67 & 0.00 & 4.73 & 3.35 & 1.67 & 0.00 \\
\hline \multirow[t]{3}{*}{ Pajek/Reuters911 } & 2304 & 2543 & 3338 & 4010 & 2497 & 2666 & 3338 & 4010 \\
\hline & $+\quad 27.48$ & $+\quad 12.81$ & +30.64 & 0.00 & 14.46 & $+\quad 61.28$ & $+\quad 31.33$ & 0.00 \\
\hline & $-\quad 12.03$ & $-\quad 54.54$ & $-\quad 17.70$ & 0.00 & 38.27 & 35.40 & $-\quad 17.70$ & 0.00 \\
\hline \multirow[t]{3}{*}{ "AG-Monien/diag } & $\overline{-1268}$ & $\overline{-1243}$ & $\overline{-1001}$ & $\overline{-951.9}$ & $\overline{-1037}$ & $\overline{-1050}$ & $\overline{-1001}$ & $\overline{-951.9}$ \\
\hline & $+\quad 86.25$ & $+\quad 96.91$ & 2.28 & 0.00 & 0.00 & 4.56 & 2.28 & 0.00 \\
\hline & 0.00 & 0.00 & - 11.31 & 0.00 & $-\quad 27.06$ & $-\quad 22.62$ & - 11.31 & 0.00 \\
\hline \multirow[t]{3}{*}{ AG-Monien/netz4504 } & -704.4 & -588.6 & -554.5 & -403.8 & -670.0 & -705.2 & -554.5 & -403.8 \\
\hline & $+\quad 10.32$ & $+\quad 31.02$ & +31.93 & 0.00 & $+\quad 56.44$ & $+\quad 63.87$ & $+\quad 30.95$ & 0.00 \\
\hline & - 67.43 & $-\quad 14.70$ & $-\quad 10.55$ & 0.00 & $-\quad 30.87$ & - 21.11 & $-\quad 10.55$ & 0.00 \\
\hline \multirow[t]{3}{*}{ AG-Monien/stufe-10 } & -15110 & -15099 & -10582 & -10531 & -10626 & -10632 & -10582 & -10531 \\
\hline & 3838 & 3877 & 5.82 & 0.00 & 9.42 & $+\quad 11.64$ & 5.82 & 0.00 \\
\hline & 0.00 & 0.00 & 1.61 & 0.00 & 4.11 & 3.22 & 1.61 & 0.00 \\
\hline \multirow[t]{3}{*}{ AG-Monien/ukerbe1 } & -2247 & -1872 & -1696 & -1242 & -2063 & -2150 & -1696 & -1242 \\
\hline & $+\quad 72.41$ & $+\quad 56.07$ & $+\quad 49.51$ & 0.00 & $+\quad 45.99$ & $+\quad 99.02$ & $+\quad 49.51$ & 0.00 \\
\hline & $-\quad 24.27$ & $-\quad 29.96$ & - 11.86 & 0.00 & $-\quad 58.92$ & $-\quad 23.72$ & -13.18 & 0.00 \\
\hline \multirow[t]{3}{*}{ DIMACS10/kron_g500-logn16 } & 9.01 & 18.71 & 317.6 & 606.1 & 13.86 & 29.11 & 317.6 & 606.1 \\
\hline & 1.39 & 1.39 & 0.00 & 0.00 & 0.00 & 0.00 & 0.00 & 0.00 \\
\hline & 6.24 & 9.01 & 6.93 & 0.00 & 8.32 & - 13.86 & 6.93 & 0.00 \\
\hline \multirow[t]{3}{*}{ Newman/as-22july06 } & -5523 & -4943 & -4550 & -3741 & -5354 & -5358 & -4550 & -3741 \\
\hline & $+\quad 14.71$ & 0.00 & 8.70 & 0.00 & $+\quad 16.21$ & $+\quad 17.39$ & 7.68 & 0.00 \\
\hline & - 81.90 & $-\quad 155.6$ & -108.8 & 0.00 & $-\quad 203.4$ & $-\quad 217.7$ & $-\quad 109.7$ & 0.00 \\
\hline \multirow[t]{3}{*}{ Pajek/Erdos981 } & -383.9 & -374 . & -371.0 & -361.2 & -380.9 & -380.9 & -371.0 & -361.2 \\
\hline & $+\quad 13.76$ & 6.67 & 5.12 & 0.00 & 11.35 & $+\quad 10.25$ & 5.06 & 0.00 \\
\hline & $-\quad 1.42$ & 4.65 & 1.17 & 0.00 & 7.15 & 2.33 & 1.17 & 0.00 \\
\hline \multirow[t]{3}{*}{ SNAP/Oregon-1 } & -2828 & -2590 & -2317 & -1884 & -2725 & -2750 & -2318 & -1884 \\
\hline & $+\quad 41.40$ & $+\quad 30.97$ & $+\quad 16.48$ & 0.00 & $+\quad 23.81$ & $+\quad 32.96$ & $+\quad 17.26$ & 0.00 \\
\hline & $-\quad 36.37$ & $-\quad 56.59$ & $-\quad 52.97$ & 0.00 & $-\quad 74.21$ & -105.93 & $-\quad 55.19$ & 0.00 \\
\hline
\end{tabular}


Table 6.4: Times (in seconds) for each variant.

\begin{tabular}{l|c|c|c|c||c|c|c|c}
\hline Problem & I & II & III & IV & V & VI & VII & VIII \\
\hline GHS_indef/aug2d & 0.043 & 0.043 & 0.054 & 0.099 & 0.045 & 0.045 & 0.056 & 0.104 \\
GHS_indef/aug2dc & 0.046 & 0.045 & 0.056 & 0.122 & 0.048 & 0.047 & 0.058 & 0.126 \\
GHS_indef/aug3d & 0.016 & 0.015 & 0.017 & 0.027 & 0.017 & 0.016 & 0.018 & 0.029 \\
GHS_indef/dtoc & 0.958 & 0.957 & 1.135 & 2.041 & 0.960 & 0.959 & 1.136 & 2.043 \\
Newman/astro-ph & 0.081 & 0.123 & 0.123 & 0.124 & 0.140 & 0.224 & 0.223 & 0.224 \\
Newman/cond-mat & 0.022 & 0.026 & 0.026 & 0.026 & 0.034 & 0.041 & 0.041 & 0.043 \\
Newman/cond-mat-2003 & 0.098 & 0.140 & 0.139 & 0.141 & 0.173 & 0.248 & 0.247 & 0.247 \\
Newman/cond-mat-2005 & 0.185 & 0.249 & 0.244 & 0.251 & 0.318 & 0.458 & 0.459 & 0.454 \\
Newman/hep-th & 0.009 & 0.008 & 0.008 & 0.009 & 0.013 & 0.013 & 0.013 & 0.014 \\
Newman/netscience & 0.001 & 0.001 & 0.001 & 0.001 & 0.001 & 0.001 & 0.001 & 0.001 \\
Pajek/Reuters911 & 0.183 & 0.113 & 0.846 & 1.910 & 0.196 & 0.127 & 0.860 & 1.948 \\
\hline AG-Monien/diag & 0.011 & 0.011 & 0.006 & 0.021 & 0.011 & 0.011 & 0.006 & 0.021 \\
AG-Monien/netz4504 & 0.002 & 0.001 & 0.001 & 0.002 & 0.002 & 0.001 & 0.002 & 0.002 \\
AG-Monien/stufe-10 & 0.287 & 0.283 & 0.172 & 0.540 & 0.333 & 0.329 & 0.219 & 0.589 \\
AG-Monien/ukerbe1 & 0.005 & 0.004 & 0.004 & 0.006 & 0.005 & 0.005 & 0.005 & 0.007 \\
DIMACS10/kron_g500-logn16 & 8.013 & 5.024 & 52.31 & 133.7 & 8.382 & 5.327 & 52.55 & 138.1 \\
Newman/as-22july06 & 0.026 & 0.034 & 0.098 & 0.179 & 0.027 & 0.036 & 0.100 & 0.186 \\
Pajek/Erdos981 & 0.001 & 0.001 & 0.001 & 0.001 & 0.001 & 0.001 & 0.001 & 0.001 \\
SNAP/Oregon-1 & 0.009 & 0.011 & 0.027 & 0.048 & 0.010 & 0.012 & 0.028 & 0.050 \\
\hline
\end{tabular}

We next consider symmetric matchings using the following variants that are the symmetric counterparts of I to IV:

V: Algorithm 3 (Duff and Pralet).

VI: Algorithm 3 with symmetric preprocessing.

VII: Algorithm 5 with a single pass on $\hat{A}$. That is, step 4 is replaced by the following single statement:

4. Apply Algorithm 4 to $\hat{A}$ to improve the matching $\sigma$ and $\mathcal{I} \times \mathcal{J}$,

and, at step 6 , use $A_{\mathcal{J} \times \mathcal{J}}$.

VIII: Algorithm 5 (Hogg and Scott).

Results are given in columns 6 to 9 of Table 6.3, with timings in Table 6.4. Each variant V-VIII is again dependent on the initial ordering. Note that only variants I and V use unsymmetric preprocessing (2.1), while the remainder use symmetric preprocessing (4.1).

Looking at I and V, we can see how the Duff and Pralet strategy that imposes symmetry affects the performance. In some cases (including GHS_indef/dtoc) it leads to a poorer matching but it can also give a better result. These mixed findings are because neither is optimal. Comparing V and VI allows us to assess how symmetric preprocessing effects the Duff and Pralet algorithm. We see that it can be beneficial but, disappointingly, the benefit is relatively small and there is no consistent advantage.

There are two key differences between VI (Duff and Pralet with symmetric preprocessing) and VII (Hogg and Scott applied to $A$ only). The first is the action taken when no $\sigma$-augmenting path is found. Variant VI terminates whereas VII continues to look to alter the column set $\mathcal{J}$ in such as way as to increase the product of the matched entries. Secondly, having chosen $\mathcal{I}$, variant VI uses $A_{\mathcal{I} \times \mathcal{I}}$. However, in VII, we interchange the roles of $\mathcal{I}$ and $\mathcal{J}$ and use $A_{\mathcal{J} \times \mathcal{J}}$. This is because it is $\mathcal{J}$ that has been constructed by Algorithm 4 to be optimal (if we were to use $A_{\mathcal{I} \times \mathcal{I}}$, variant VII would reduce to variant VI since both give the same set $\mathcal{I})$. Note that we experimented with modifying VI to also work with $\mathcal{J} \times \mathcal{J}$ but, as neither the $\mathcal{I}$ nor the $\mathcal{J}$ constructed by the Duff and Pralet approach is optimal, this was not beneficial.

A comparison of variants VII and VIII demonstrates how the matching is improved by applying Algorithm 4 to both $A_{\mathcal{I} \times \mathcal{I}}$ and $A_{\mathcal{I} \times \mathcal{I}}^{T}$. As before, using $A_{\mathcal{I} \times \mathcal{I}}^{T}$ offers a consistent advantage (with the 
largest benefit generally being for problems with a large rank deficiency) but for some problems the time overhead to achieve this can be substantial.

Since IV produces the optimal value of the objective function, comparing it with VIII illustrates the effect of imposing symmetry. For each of our test problems, the symmetry constraint leads to no decrease in the objective function value (and the VIII results are seen not to be dependent on the initial ordering). Similarly, comparing III and VII illustrates the effect of imposing symmetry when only $A$ is used. For some examples, the objective function value decreases when symmetry is imposed but the improvements are very small. From Table 6.4, we see that the symmetry time overhead is generally less than 10 per cent, although it can be close to 100 per cent (for example, problem Newman/cond-mat).

\section{Concluding remarks}

In this paper, we have shown how to modify earlier work on weighted matchings to obtain optimal weighted matchings for rank-deficient matrices. Our approach is applicable to both unsymmetric and symmetric matrices but our main emphasis is on computing optimal symmetric matchings. We have modified the approach of Duff and Pralet for symmetric matrices in three key ways. Firstly, we have introduced a symmetric preprocessing to retain symmetry. Secondly, we implicitly work with a modified matrix $A(\epsilon)$ that allows us to circumvent the problem of some rows/columns being unmatched in the structurally singular case. Finally, by working with both $A$ and $A^{T}$ we have developed an implementation that is practical for large problems. We have shown, using examples from real applications, that while our approach can involve a considerable time overhead, the benefit for some problems is a significant improvement in the quality of the matching. We remark that it is not possible to know a priori how far the matching returned by the Duff and Pralet algorithm is from optimal and hence how much the matching will be improved by our modified approach.

\section{Acknowledgements}

We would like to thank Iain Duff for commenting on a draft of this paper.

\section{References}

[1] T. Davis And Y. Hu, The University of Florida Sparse Matrix Collection, ACM Transactions on Mathematical Software, 38 (2011). Article 1, 25 pages.

[2] E. W. Dijkstra, A note on two problems in connection with graphs, Numerische Mathematik, 1 (1959), pp. 269-271.

[3] I. Duff And J. Gilbert, Maximum-weighted matchingand block pivoting for symmetric indefinite systems. in Abstract book of Householder Symposium XV, June 17-21, 2002, pp.73-75.

[4] I. Duff And J. Koster, On algorithms for permuting large entries to the diagonal of a sparse matrix, SIAM J. on Matrix Analysis and Applications, 22 (2001), pp. 973-996.

[5] I. Duff And S. Pralet, Strategies for scaling and pivoting for sparse symmetric indefinite problems, SIAM J. on Matrix Analysis and Applications, 27 (2005), pp. 313 - 340.

[6] HSL, A collection of Fortran codes for large-scale scientific computation, 2011. http://www.hsl.rl.ac.uk/.

[7] H. Kunn, The Hungarian method for the assignment problem, Naval Research Logistics Quarterly, 2 (1955), pp. 83-97.

[8] M. Olschowka And A. Neumaier, A new pivoting strategy for Gaussian elimination, Linear Algebra and Its Applications, 240 (1996), pp. 131-151. 